\title{
The influence of olfactory dysfunction on risk of malnutrition is mediated by depressive symptoms in cancer patients undergoing chemotherapy
}

\author{
Yanfeng Lin, Xiaocong Jiang, Xinmei Huang, Yifan Lv \\ Department of Internal Medicine, Huizhou Municipal Central Hospital, Huizhou, China \\ Contributions: (I) Conception and design: Y Lv, Y Lin; (II) Administrative support: Y Lv; (III) Provision of study materials or patients: Y Lin, X Jiang, \\ X Huang, Y Lv; (IV) Collection and assembly of data: Y Lin, X Jiang, X Huang; (V) Data analysis and interpretation: Y Lin, Y Lin; (VI) Manuscript \\ writing: All authors; (VII) Final approval of manuscript: All authors. \\ Correspondence to: Yifan Lv. Department of Internal Medicine, Huizhou Municipal Central Hospital, 41 Helingbei Road, Huizhou 516000, China. \\ Email: YifanLv@21cn.com.
}

\begin{abstract}
Backgroundk Malnutrition is common and detrimental in cancer patients undergoing chemotherapy. There are close associations between olfactory dysfunction, depression, and malnutrition, but how they correlate in cancer patients undergoing chemotherapy remains unclear.

Methods: Two hundred and one cancer patients undergoing chemotherapy were recruited to this study. Their risk of malnutrition was assessed using the Simplified Nutritional Appetite Questionnaire (SNAQ); odor identification was assessed by Sniffin' Sticks test; self-measurement of olfaction was assessed by Selfreported Mini Olfactory Questionnaire (Self-MOQ); and depressive symptoms were assessed using the Beck Depression Inventory (BDI). Correlation analyses and mediation analyses were used to explore the relationships between olfaction, depression, and risk of malnutrition.

Results: The SNAQ score was negatively correlated with the Self-MOQ score and BDI score, and positively correlated with body mass index (BMI) scores and odor identification. The Self-MOQ score was negatively correlated with odor identification and positively correlated with BDI scores, and the duration of chemotherapy was negatively correlated with odor identification. Mediation analyses suggested that BDI scores exhibited a partial mediation effect on the relationship between Self-MOQ score and SNAQ scores.

Conclusions: The influence of olfactory dysfunction on risk of malnutrition is mediated by depressive symptoms in cancer patients undergoing chemotherapy. Early intervention of olfactory dysfunction and depressive symptoms may be helpful in reducing the risk malnutrition in cancer patients undergoing chemotherapy.
\end{abstract}

Keywords: Olfaction; malnutrition; depression; cancer; chemotherapy

Submitted Aug 01, 2021. Accepted for publication Oct 19, 2021.

doi: 10.21037/apm-21-2221

View this article at: https://dx.doi.org/10.21037/apm-21-2221

\section{Introduction}

Malnutrition is a prognostic factor related to morbidity, mortality, and costs of care in cancer patients undergoing chemotherapy (1), and it is associated with negative clinical outcomes including poorer quality of life (2), reduced general status (3), and poorer survival (4), decreased bone mass (5), delayed recovery of surgery, high hospitalization and readmission rates, and immune dysfunction (6). In addition, malnutrition is common, with an incidence of $45-65 \%$ in cancer patients. Therefore, among patients undergoing chemotherapy, early identification and intervention of those at higher risk of malnutrition is 
important as it can significantly reduce the disease burden the increase patients' quality of life.

Olfactory dysfunction is closely related to nutritional changes (7), and it has been reported as common in cancer patients undergoing chemotherapy (8). Individuals with olfactory dysfunction usually complain that food is not as flavorful and enjoyable as before, and their cooking and eating habits may be disrupted by their inability to perceive food-related odors (9). During the administration of cytotoxic chemotherapy, the chemosensory systems are exposed to more change than other sensory systems (10), which may be due to the short life span of olfactory receptor cells and their frequent renewal, which make them more assailable to chemotherapy drugs $(11,12)$.

Depression is one of the most common symptoms in cancer patients undergoing chemotherapy, with a prevalence of $8-24 \%$ (13). Interestingly, depressive symptoms are also closely related to malnutrition and olfactory dysfunction. On the one hand, patients with depression are more likely to exhibit loss of appetite, decreased food intake, meal skipping, and disordered eating, which can lead to poor nutritional outcomes (14). Oxidative and nitrosative stress, inflammation, a decrease in antioxidant levels, and many other pathways may be the underlying mechanism of depression in malnutrition (15). On the other hand, the olfactory system strongly overlaps with those related to emotional modulation (such as the amygdala, orbitalfrontal cortex, and insular) (16), and patients with olfactory dysfunction are frequently accompanied with depressive symptoms (17). In addition, olfactory training may be useful in reducing depressive symptoms in elderly individuals (18).

In sum, olfactory dysfunction, malnutrition, and depression are closely associated with each other, but their relationships in cancer patients undergoing chemotherapy remain unclear. Based on the previous evidence, we hypothesized that the increasing risk of malnutrition by olfactory dysfunction is mediated by depressive symptoms in cancer patients undergoing chemotherapy. The present study aimed to explore the relationship between olfactory dysfunction, risk of malnutrition, and depressive symptoms in cancer patients undergoing chemotherapy by using psychophysical and neuropsychological assessments.

We present the following article in accordance with the STROBE reporting checklist (available at https://dx.doi. org/10.21037/apm-21-2221).

\section{Methods}

\section{Participants}

Two hundred and one cancer patients undergoing chemotherapy (age 55.14 \pm 10.69 years, 106 male, 95 female) were recruited from the Huizhou Municipal Central Hospital. Diagnoses were made by at least 2 doctors from the Department of Oncology. The exclusion criteria were other situations that significantly influence olfactory function, including respiratory distress or active upper respiratory/sinus infection during the time of testing, congenital or traumatic anosmia, current or recent (past 6 months) smoking, and alcohol or substance dependence. Information about height, weight, disease duration, drugs, and duration of chemotherapy was collected. Body mass index (BMI) is defined as a person's weight in kilograms divided by the square of the person's height in meters $\left(\mathrm{kg} / \mathrm{m}^{2}\right)$. The study was conducted in accordance with the Declaration of Helsinki (as revised in 2013). All participants or their legal guardians provided signed informed consent to participate in the study. This study was approved by the Ethics Committee of Huizhou Municipal Central Hospital (Huizhou, China).

\section{Assessment of risk of malnutrition}

The Simplified Nutritional Appetite Questionnaire (SNAQ) (19) was used to assess the risk of malnutrition. The SNAQ is composed of 4 items, grouped in a dingle domain. Each question presents 5 options [1-5], and the total score ranges from 4 to 20 . The lower the score is, the higher the risk for weight loss.

\section{Assessment of olfactory function}

The Sniffin' Sticks test (Burghardt, Wedel, Germany) (20) was used to assess odor identification, which involves the presentation of odors from felt-tip pens. The experimenter opened the cap for about 3 seconds, and placed the pen tip of odorized pens about $2 \mathrm{~cm}$ in front of both nostrils. Participants were asked to smell 16 common odors from the pen, and then consult the form, choosing from 4 options to name these odors. The SNAQ scores ranged from 0 to 16 points. A higher score reflected more accurate odor 
Table 1 Demographic and clinical characteristics of study sample

\begin{tabular}{lcc}
\hline \multirow{2}{*}{ Variables } & \multicolumn{2}{c}{ Total sample $(\mathrm{n}=201)$} \\
\cline { 2 - 3 } & Mean & $\mathrm{SD}$ \\
\hline Age (years) & 55.14 & 10.69 \\
$\mathrm{BMI}\left(\mathrm{kg} / \mathrm{m}^{2}\right)$ & 22.10 & 3.17 \\
Duration of illness (months) & 12.53 & 21.81 \\
Duration of chemotherapy (months) & 10.69 & 21.03 \\
SNAQ & 14.65 & 2.84 \\
Self-MOQ & 3.28 & 7.45 \\
Odor identification & 10.43 & 2.89 \\
BDI & 3.53 & 3.85 \\
\hline
\end{tabular}

BMI, body mass index; SNAQ, Simplified Nutritional Appetite Questionnaire; Self-MOQ, Self-reported Mini Olfactory Questionnaire; BDI, Beck Depression Inventory; SD, standard deviation.

identification.

The Self-reported Mini Olfactory Questionnaire (SelfMOQ) (21) was used to assess participants' self-assessment of olfaction. The Self-MOQ consists of 14 items, and the items are formulated as personal statements, which reflect complaints about olfactory problems in daily life (e.g., "I like to look around the flower shop, but I cannot smell anything"). Each question includes 4 degrees, "totally disagree" score 0 , "partially disagree" score 1, "partially agree" score 2 , and "totally agree" score 3 . The total scores range from 0 to 42 . Higher scores represent worse olfactory function.

\section{Assessment of depressive symptoms}

The Beck Depression Inventory (BDI) (22) was used to assess participants' depressive symptoms. The BDI includes 21 items, and each item has a score of $0-3$. The total score of BDI ranges from 0 to 63 , and higher scores represent more severe depressive symptoms.

\section{Statistical analysis}

The distribution of data was presented using mean \pm standard deviation (SD). Partial correlation was used to explore the relationship between disease duration, duration of chemotherapy, BMI, odor identification, Self-MOQ score, SNAQ score, and BDI scores, and controlling variable including age and gender. Mediation analyses were performed for potential variables screened in correlation analyses. The mediation model was established when the following conditions were met: (I) the independent variable (IV) had a significant effect on the dependent variable (DV); (II) the IV significantly predicted the mediator; (III) the mediator significantly affected the DV; and (IV) when the mediator was excluded in the model, the effect of the IV on the DV decreased. The PROCESS tool (https://www. processmacro.org/index.html) was used to investigate the mediation model among variables. Indirect effects were estimated with 1,000 bootstrapped samples. Moreover, the Goodman test (23) was performed to verify whether the mediating effect was significant.

\section{Results}

\section{Demographic data and score of assessment}

The demographic data, including scores of various assessments are listed in Table 1.

\section{Correlation analyses}

It was shown that BMI positively correlated with SNAQ score $(\mathrm{r}=0.171, \mathrm{P}=0.015)$ (Figure $1 A)$; duration of chemotherapy was negatively correlated with odor identification $(\mathrm{r}=-0.142, \mathrm{P}=0.045)$ (Figure $1 B)$; SNAQ score was negatively correlated with Self-MOQ score $(r=-0.239$, $\mathrm{P}=0.001)$ (Figure $1 C)$ and BDI score $(\mathrm{r}=-0.224, \mathrm{P}=0.001)$ (Figure 1D); Self-MOQ score was negatively correlated with odor identification $(\mathrm{r}=-0.294, \mathrm{P}<0.001)$ (Figure $1 E)$ and positively correlated with BDI scores $(\mathrm{r}=0.260, \mathrm{P}<0.001)$ (Figure $1 F$ ); and odor identification was positively correlated with SNAQ scores $(\mathrm{r}=0.197, \mathrm{P}=0.005)$ (Figure $1 G)$. No other significant correlation was found among different variables.

\section{Mediation analyses}

The indirect effect of Self-MOQ scores on SNAQ scores through BDI scores was $0.078(\mathrm{Z}=2.00, \mathrm{P}=0.045)$, the remaining direct effect of Self-MOQ scores on SNAQ scores was still significant, which manifested that olfactory dysfunction negatively affected nutrition condition by upregulating depressive symptom (Figure 2). No other significantly mediated effect was found. 


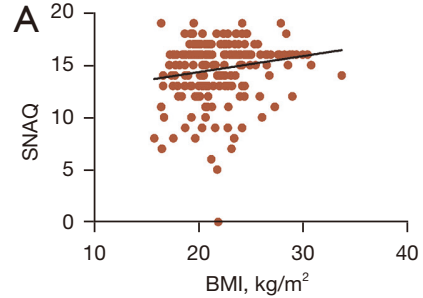

D

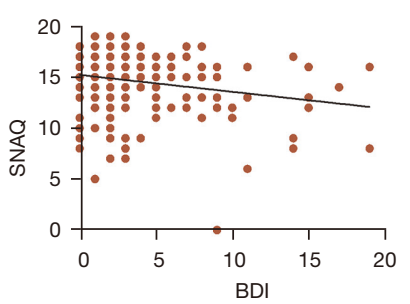

G

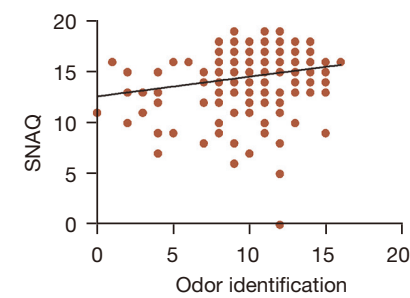

B
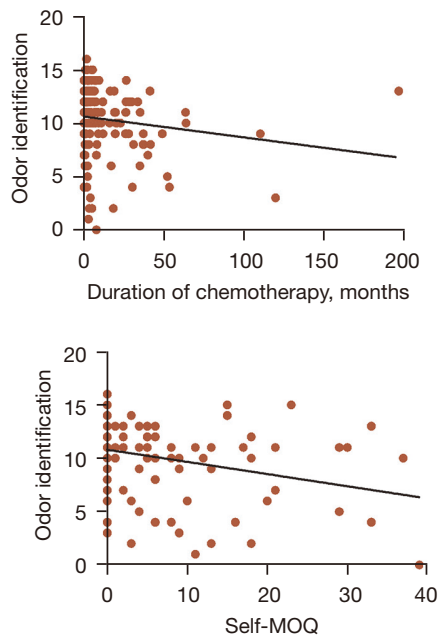

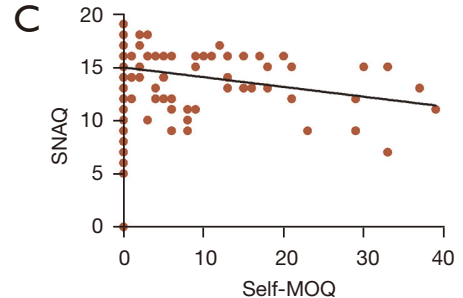

$\mathrm{F}$

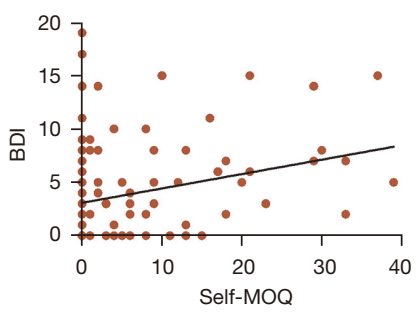

Figure 1 Correlation analyses between olfaction, depression and malnutrition. (A) BMI positively correlated with SNAQ score ( $\mathrm{r}=0.171$, $\mathrm{P}=0.015$ ); (B) duration of chemotherapy was negatively correlated with odor identification $(\mathrm{r}=-0.142, \mathrm{P}=0.045)$; (C) SNAQ score was negatively correlated with Self-MOQ score $(r=-0.239, \mathrm{P}=0.001)$; (D) SNAQ score was negatively correlated with BDI score $(\mathrm{r}=-0.224$, $\mathrm{P}=0.001)$; (E) Self-MOQ score was negatively correlated with odor identification ( $\mathrm{r}=-0.294, \mathrm{P}<0.001$ ); (F) Self-MOQ score was positively correlated with BDI scores $(r=0.260, P<0.001)$; (G) odor identification was positively correlated with SNAQ scores $(r=0.197, P=0.005)$. BMI, body mass index; SNAQ, Simplified Nutritional Appetite Questionnaire; Self-MOQ, Self-reported Mini Olfactory Questionnaire; BDI, Beck Depression Inventory.

\section{Discussion}

This was the first study to explore the mediated effect of depressive symptoms on the relationship between olfactory dysfunction and risk of malnutrition in cancer patients undergoing chemotherapy. The main results were: (I) SNAQ score was negatively correlated with Self-MOQ score and BDI score, and positively correlated with BMI scores and odor identification; (II) Self-MOQ score was negatively correlated with odor identification and positively correlated with BDI score, and duration of chemotherapy was negatively correlated with odor identification; (III) BDI score exhibited a partial mediation effect on the relationship between Self-MOQ score and SNAQ score.

The present study demonstrated that olfactory dysfunction was associated with increasing risk of malnutrition in cancer patients undergoing chemotherapy, which was consistent with previous studies (8). In addition, we found that both objective (Sniffin' Sticks) and subjective assessment (Self-MOQ) of olfactory function were associated with risk of malnutrition. Spotten et al. suggested that compared with objective measures of smell and taste function, subjective assessment may be more reliable to reflect the patient situation and predict changes in dietary behavior (24). Consistently, the current results suggested that self-assessments were strongly associated with objective assessment of odor identification, and may be more sensitive to predict risk of malnutrition. Therefore, self-assessment of olfactory dysfunction may serve as a simple and useful tool for predicting risk of malnutrition in future clinical practice.

It has been repeatedly reported that depression is associated with metabolic disturbance (25), and the current study confirmed that the more severe depressive symptoms, 


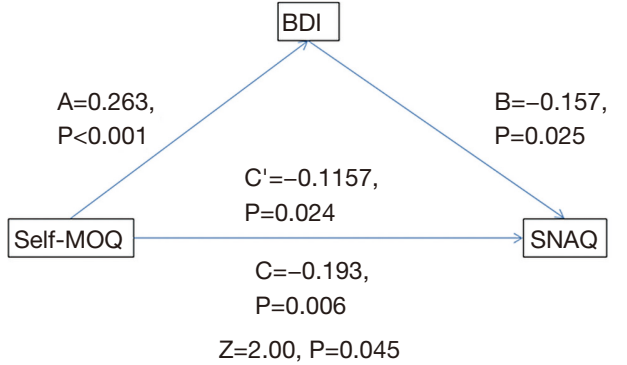

Figure 2 The relationship between Self-MOQ and SNAQ was mediated by BDI. The indirect effect of Self-MOQ scores on SNAQ scores through BDI scores was $0.078(\mathrm{Z}=2.00, \mathrm{P}=0.045)$, the remaining direct effect of Self-MOQ scores on SNAQ scores was still significant, which manifested that olfactory dysfunction negatively affected nutrition condition by upregulating depressive symptom. SNAQ, Simplified Nutritional Appetite Questionnaire; Self-MOQ, Self-reported Mini Olfactory Questionnaire; BDI, Beck Depression Inventory.

the higher risk of malnutrition. The negative correlation between SNAQ score and BDI score may be the result of meal skipping, decreased food intake, and loss of appetite. For the relationship between olfaction and depression, our results suggested that BDI scores were associated with selfassessments of olfactory dysfunction, but not objective assessment of odor identification. On the one hand, depressive symptoms are more associated with periphery dysfunction of olfactory symptoms (such as olfactory ball and olfactory receptor), and odor identification is an indicator of high-order functioning of the olfactory system (16). On the other hand, the questions used in the self-MOQ are "if you can smell something" and more associated with odor sensitivity, which reflect the peripheral function of olfactory system (21).

Considering the closed relationship between malnutrition, depression, and olfaction, a mediation analysis was used to further explore their interaction. We found that depressive symptoms exhibited a partial mediation effect on the relationship between olfactory dysfunction and risk of malnutrition, suggesting that the association between olfactory dysfunction and risk of malnutrition became weaker after removing the effect of depressive symptoms. This implied that olfactory dysfunction may not only increase the risk of malnutrition directly, but also increase depressive symptoms indirectly. Future follow-up studies are needed to confirm if olfactory dysfunction could increase the risk of malnutrition by increasing depressive symptoms, and explore whether improving olfaction and decreasing depressive symptoms could reduce the risk of malnutrition.

There were several limitations in the present study. First, the analyses were based on cross-sectional design, and the relationship between risk of malnutrition, depressive symptoms, and olfactory dysfunction needs to be further confirmed by follow-up study. Second, olfactory function was measured by self-assessment and odor identification in the present study. Future research is needed to explore whether odor threshold and odor discrimination may exhibit a similar association with risk of malnutrition and depressive symptoms. Last, the present sample involved a combination of different kinds of cancer, and whether the associations between risk of malnutrition, depressive symptoms, and olfactory dysfunction may be different in various cancers needs to be further explored.

\section{Conclusions}

The risk of malnutrition is associated with olfactory dysfunction and more severe depressive symptoms in cancer patients undergoing chemotherapy. Additionally, the influence of olfactory dysfunction on risk of malnutrition is mediated by depressive symptoms. Early intervention of olfactory dysfunction and depressive symptoms may be helpful in reducing the risk of malnutrition in cancer patients undergoing chemotherapy.

\section{Acknowledgments}

Funding: None.

\section{Footnote}

Reporting Checklist: The authors have completed the STROBE reporting checklist. Available at https://dx.doi. org/10.21037/apm-21-2221

Data Sharing Statement: Available at https://dx.doi. org/10.21037/apm-21-2221

Conflicts of Interest: All authors have completed the ICMJE uniform disclosure form (available at https://dx.doi. org/10.21037/apm-21-2221). The authors have no conflicts of interest to declare.

Ethical Statement: The authors are accountable for all 
aspects of the work in ensuring that questions related to the accuracy or integrity of any part of the work are appropriately investigated and resolved. The study was conducted in accordance with the Declaration of Helsinki (as revised in 2013). All participants or their legal guardians provided signed informed consent to participate in the study. This study was approved by the Ethics Committee of Huizhou Municipal Central Hospital (Huizhou, China).

Open Access Statement: This is an Open Access article distributed in accordance with the Creative Commons Attribution-NonCommercial-NoDerivs 4.0 International License (CC BY-NC-ND 4.0), which permits the noncommercial replication and distribution of the article with the strict proviso that no changes or edits are made and the original work is properly cited (including links to both the formal publication through the relevant DOI and the license). See: https://creativecommons.org/licenses/by-nc-nd/4.0/.

\section{References}

1. Kiss NK, Krishnasamy M, Isenring EA. The effect of nutrition intervention in lung cancer patients undergoing chemotherapy and/or radiotherapy: a systematic review. Nutr Cancer 2014;66:47-56.

2. Isenring E, Bauer J, Capra S. The scored Patientgenerated Subjective Global Assessment (PG-SGA) and its association with quality of life in ambulatory patients receiving radiotherapy. Eur J Clin Nutr 2003;57:305-9.

3. Nourissat A, Vasson MP, Merrouche Y, et al. Relationship between nutritional status and quality of life in patients with cancer. Eur J Cancer 2008;44:1238-42.

4. Ross PJ, Ashley S, Norton A, et al. Do patients with weight loss have a worse outcome when undergoing chemotherapy for lung cancers? Br J Cancer 2004;90:1905-11.

5. Fávaro-Moreira NC, Krausch-Hofmann S, Matthys C, et al. Risk Factors for Malnutrition in Older Adults: A Systematic Review of the Literature Based on Longitudinal Data. Adv Nutr 2016;7:507-22.

6. Ahmed T, Haboubi N. Assessment and management of nutrition in older people and its importance to health. Clin Interv Aging 2010;5:207-16.

7. Croy I, Nordin S, Hummel T. Olfactory disorders and quality of life--an updated review. Chem Senses 2014;39:185-94.

8. Drareni K, Dougkas A, Giboreau A, et al. Relationship between food behavior and taste and smell alterations in cancer patients undergoing chemotherapy: A structured review. Semin Oncol 2019;46:160-72.

9. Aschenbrenner K, Hummel C, Teszmer K, et al. The influence of olfactory loss on dietary behaviors. Laryngoscope 2008;118:135-44.

10. Yakirevitch A, Talmi YP, Baram Y, et al. Effects of cisplatin on olfactory function in cancer patients. Br J Cancer 2005;92:1611-3.

11. Lee SJ, Depoortere I, Hatt H. Therapeutic potential of ectopic olfactory and taste receptors. Nat Rev Drug Discov 2019;18:116-38.

12. Barlow LA, Klein OD. Developing and regenerating a sense of taste. Curr Top Dev Biol 2015;111:401-19.

13. Linden W, Vodermaier A, Mackenzie R, et al. Anxiety and depression after cancer diagnosis: prevalence rates by cancer type, gender, and age. J Affect Disord 2012;141:343-51.

14. Ghimire S, Baral BK, Pokhrel BR, et al. Depression, malnutrition, and health-related quality of life among Nepali older patients. BMC Geriatr 2018;18:191.

15. Maes M, Galecki P, Chang YS, et al. A review on the oxidative and nitrosative stress (O\&NS) pathways in major depression and their possible contribution to the (neuro)degenerative processes in that illness. Prog Neuropsychopharmacol Biol Psychiatry 2011;35:676-92.

16. Croy I, Hummel T. Olfaction as a marker for depression. J Neurol 2017;264:631-8.

17. Chen B, Benzien C, Faria V, et al. Symptoms of Depression in Patients with Chemosensory Disorders. ORL J Otorhinolaryngol Relat Spec 2021;83:135-43.

18. Birte-Antina W, Ilona C, Antje H, et al. Olfactory training with older people. Int J Geriatr Psychiatry 2018;33:212-20.

19. Lau S, Pek K, Chew J, et al. The Simplified Nutritional Appetite Questionnaire (SNAQ) as a Screening Tool for Risk of Malnutrition: Optimal Cutoff, Factor Structure, and Validation in Healthy Community-Dwelling Older Adults. Nutrients 2020;12:2885.

20. Kobal G, Hummel T, Sekinger B, et al. "Sniffin' sticks": screening of olfactory performance. Rhinology 1996;34:222-6.

21. Zou LQ, Linden L, Cuevas M, et al. Self-reported mini olfactory questionnaire (Self-MOQ): A simple and useful measurement for the screening of olfactory dysfunction. Laryngoscope 2020;130:E786-90.

22. Richter P, Werner J, Heerlein A, et al. On the validity of the Beck Depression Inventory. A review. Psychopathology 1998;31:160-8.

23. Goodman LA. On the exact variance of products. J Am 
Stat Assoc 1960:708-13.

24. Spotten LE, Corish CA, Lorton CM, et al. Subjective and objective taste and smell changes in cancer. Ann Oncol 2017;28:969-84.

25. Milaneschi Y, Simmons WK, van Rossum EFC, et al.

Cite this article as: Lin Y, Jiang X, Huang X, Lv Y. The influence of olfactory dysfunction on risk of malnutrition is mediated by depressive symptoms in cancer patients undergoing chemotherapy. Ann Palliat Med 2021;10(10):10600-10606. doi: 10.21037/apm-21-2221
Depression and obesity: evidence of shared biological mechanisms. Mol Psychiatry 2019;24:18-33.

(English Language Editor: J. Jones) 